\title{
Long-Term Results of Simple Enucleation for the Treatment of Small Renal Cell Carcinoma
}

\author{
Ambrosi Pertia, Lauri Managadze \\ National Center of Urology, Tbilisi, Georgia
}

\begin{abstract}
Objective: We have analyzed our institutional experience with simple enucleation for the treatment of small renal tumors for elective indications.

Materials and Methods: A total of 30 patients underwent elective nephron-sparing surgery (NSS) from May 1997 to January 2001. All patients underwent NSS by means of enucleation. The tumor bed was coagulated carefully for haemostatic and partly for oncological reasons. Median follow-up was 71 months (range: 49-91 months).

Results: Pathological review according to the 2002 TNM classification showed that $70 \%$ (21 of 30) of tumors were pT1a, $26.7 \%$ (8 of 30) pT1b and $3.3 \%$ (1 of 30) pT3a. Median tumor size was $3.7 \mathrm{~cm}$. (range: $3.0-5.5 \mathrm{~cm}$ ). There was no perioperative mortality (within the first 30 days). Bleeding had not been recorded during perioperative period. Urinary leakage was observed in 1 patient (3.3\%). No case of local recurrence was observed. Five and 7-year cumulative survival was $96.6 \%$ and $93.3 \%$, respectively. Five and 7 -year cancer specific survival was $100 \%$ and $96.5 \%$, respectively.

Conclusions: Simple tumor enucleation is a safe and acceptable approach for elective NSS. It provides excellent long-term progression-free and cancer specific survival rates, and is not associated with an increased risk of local recurrence compared to partial nephrectomy.
\end{abstract}

Key words: renal cell carcinoma; surgery; postoperative complications; survival rate Int Braz. J Urol. 2006; 32: 640-7

\section{INTRODUCTION}

Nephron sparing surgery (NSS) was initially used in the treatment of renal cell carcinoma (RCC) only for absolute and relative indications $(1,2)$. The widespread use of modern radiological modalities substantially changed clinical presentation of renal tumors in recent decades. Currently, more than onehalf of all patients with surgically resectable renal tumors are detected incidentally $(2,3)$. In the patients with normal contralateral kidney, NSS became a standard method of treatment. Several trials have shown survival rates similar to those obtained with radical surgery for low stage, low-grade lesions with less than $4 \mathrm{~cm}$ of size $(2,4-6)$.

Local recurrence is the major drawback of NSS mostly due to incomplete resection of the primary tumor. Thus, tumor excision without leaving residual malignant tissue in the renal remnant is very important. However, the optimal margin that should be resected during NSS is still controversial. The recommended minimal size of resected parenchyma in NSS varies from investigator to investigator and includes $0.5 \mathrm{~cm}$ (5), $1 \mathrm{~cm}(6)$, "a rim" of normal ap- 
pearing parenchyma (7), and even simple enucleation $(6,8,9)$. Several recent studies have shown that a minimal layer of healthy parenchyma is quite enough without compromising oncological outcome. The simple enucleation technique, which was previously described as treatment of choice for benign kidney tumors like angiomyolipoma, was also used for the treatment of small RCC by some authors. To our knowledge, only few studies have been conducted during the last decade assessing efficacy of enucleation in $\operatorname{RCC}(6,8,9)$. In the present study, we evaluated retrospectively 30 cases of NSS performed at our institution for elective indications.

\section{MATERIALS AND METHODS}

Thirty patients underwent NSS for elective indications From May 1997 to January 2001 (Table1). There were 19 (63.3\%) males, and 11 (36.7\%)

Table 1 - Clinicopathological characteristics of $30 \mathrm{pa}$ tients.

Pte. Age at Surgery (years)

\begin{tabular}{lc} 
Median (range) & $49(37-68)$ \\
Pathological tumor size (cm) & \\
Median (range) & $3.7(3.0-5.5)$ \\
Gender & $19(63.3 \%)$ \\
N Male (\%) & $11(36.7 \%)$ \\
N Female (\%) & $27(90 \%)$ \\
N Tumorsincidentally discovered (\%) \\
N 2003 tumor stage (\%) \\
pT1a & $21(70 \%)$ \\
pT1b & $8(26.7 \%)$ \\
pT3a & $1(3.3 \%)$ \\
N Nuclear grade (\%) & \\
G1 & $12(40 \%)$ \\
G2 & $15(50 \%)$ \\
G3 & $3(10 \%)$ \\
N Histological subtype (\%) & \\
Clear cell & $22(73.3 \%)$ \\
Papillary & $5(16.7 \%)$ \\
Chromophobe & $2(6.7 \%)$ \\
Cystic RCC & $1(3.3 \%)$ \\
N Disease progression (\%) & $1(3.3 \%)$ \\
Median follow-up in months (range) & $71(49-91)$ \\
\hline
\end{tabular}

females. The median age was 49 years (range: 37 68 years). Left side tumor was detected in $18(60 \%)$ cases and right side in $12(40 \%)$ cases. The tumor was in the upper pole in 11 cases $(36.7 \%)$, mid kidney in $7(23.3 \%)$ and lower pole in $12(40 \%)$. All tumors were located peripherally (defined as peripherally located and enveloped by cortical parenchyma, without extension into the renal sinus). At diagnosis $27(90 \%)$ tumors were detected incidentally, whilst 3 $(10 \%)$ were associated with microscopic hematuria. All patients were evaluated carefully preoperatively to exclude the presence of distant metastases. Preoperative evaluation included ultrasonography of the kidney, CT of the abdomen and chest X-ray in all patients. Renal function was assessed by means of serum creatinine level.

All patients were operated through extraperitoneal, extrapleural incision above the 12th rib. The kidney was completely mobilized to exclude the presence of satellite tumors. Peritumoral fat was left in situ. A sharp incision on the renal capsule was performed 2 to $3 \mathrm{~mm}$ away from the tumor margin. The renal pedicle was completely isolated and the renal artery was clamped just before beginning the incision on the renal capsule. The venous clamping was not used in any case. To reduce the outcomes of renal ischemia vigorous hydration, mannitol infusion before the arterial clamping and renal hypothermia with ice was adopted in all cases. Tumors were enucleated without a layer of normal parenchyma. During the enucleation, a cleavage plane between pseudocapsule and normal parenchyma was created by means of scissors, without blunt finger dissection. All tumors presented a real pseudocapusle, which facilitated the enucleation. Tumor bed was inspected very carefully. Intraoperative frozen section of tumor bed was performed routinely in all cases. The results of frozen section were negative in all cases. The data of the patients who underwent nephrectomy due to positive margins found during frozen sections, were not included in the study. The visible bleeding vessels and opened calices were closed using running sutures. Finally, tumor bed was coagulated carefully for haemostatic and partly for oncological reasons. The coagulation was performed by means of diathermy spray coagulation. We did not wait for the 
intraoperative frozen section under renal ischemia and went forward with the next steps of the operation. The parenchymal defect was closed using absorbable interrupted sutures. In case of large capsular defect, it was covered with free peritoneal graft.

Pathological tumor staging was performed according to the 2002 TNM staging system (10) and nuclear grade was assigned according to Fuhrman's grading system (11). The removed tumor specimen was always inspected by pathologists and the surgical margins were inked.

The patients were followed with renal functional tests, chest $\mathrm{x}$-ray, abdominal ultrasound or CT scan every 3 months during the first year, once in 6 months for the next two years and annually thereafter. In terms of statistical analysis, the probability of cumulative and cancer-specific survival was estimated by the Kaplan-Meier method using the whole number of events.

\section{RESULTS}

Twenty one out of 30 tumors were pT1a (70\%), 8 were pT1b (26.7\%) and 1 was pT3a (3.3 $\%$ ). The median tumor size was $3.7 \mathrm{~cm}$. (range: 3.0 $5.5 \mathrm{~cm}$ ). Final pathological evaluation did not reveal any case of tumor extension out of the inked area of the surgical specimens. Grade1 was diagnosed in 12 (40\%) cases, Grade 2 in $15(50 \%)$ cases and Grade 3 in $3(10 \%)$ cases. Pathological T3a case was confirmed by the microinvasion of the surrounding perirenal fat. Histological classification revealed 22 clear cell $(73.3 \%), 5$ papillary $(16.7 \%), 2$ chromophobe $(6.7 \%)$ and 1 cystic (3.3\%) RCC.

The median time of renal ischemia was 22 minutes (range: $18-35 \mathrm{~min}$.). No perioperative mortality and/or serious general complications (myocardial infarction, deep venous thrombosis etc) were observed. No wound infection was observed during the early postoperative period. Bleeding had not been recorded during early postoperative (within the first 30 days) period. Urinary leakage was observed in 1 patient $(3.3 \%)$ and patient required double-J stenting. Median hospital stay was 6 days (range 4 - 15). The median follow-up was 71 months (range: 49 - 91 months). No case of local recurrence was observed. Renal functions were stable in all patients during follow-up period. Renal function remained stable in all patients with a median postoperative creatinine level of $0.9 \mathrm{mg} / \mathrm{dL}$ (range: $0.7-1.4 \mathrm{mg} / \mathrm{dL}$ ).

One patient with pT1b, G3 disease developed osseous metastases and died 81 months postoperatively. The tumor size in this patient was $45 \mathrm{~mm}$. One patient died for kidney unrelated cancer reasons without evidence of tumor recurrence.

The remaining 28 patients are alive without evidence of disease recurrence at the last checkup. Five and 7-year cumulative survival was $96.6 \%$ and 93.3\%, respectively (Figure-1). Five and 7-year cancer specific survival was $100 \%$ and $96.5 \%$, respectively (Figure-2).

\section{COMMENTS}

Local recurrence is the major drawback of NSS mostly due to incomplete resection of the primary tumor, occult multicentric disease, or the development of new primary or metastatic cancer in the

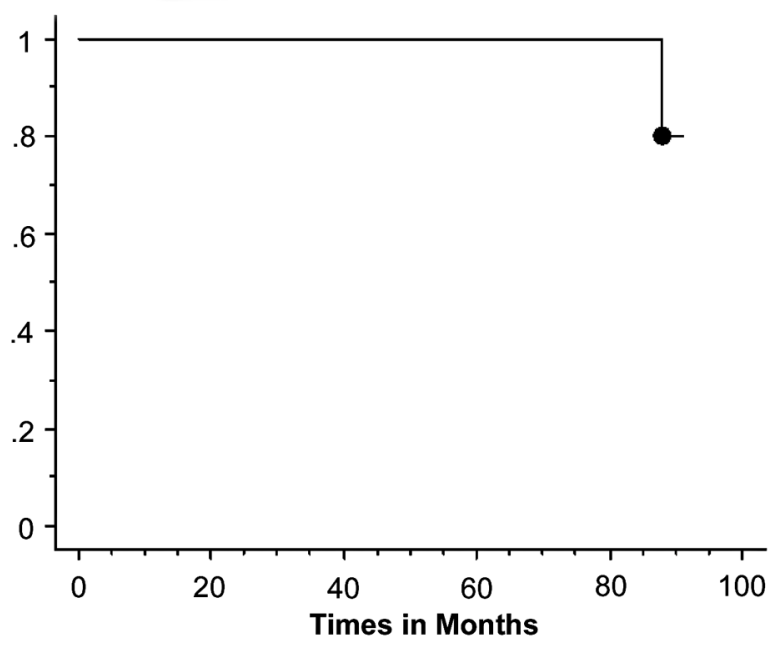

Figure 1 - Cancer-specific survival rate in patients undergoing nephron-sparing surgery by simple enucleation. 


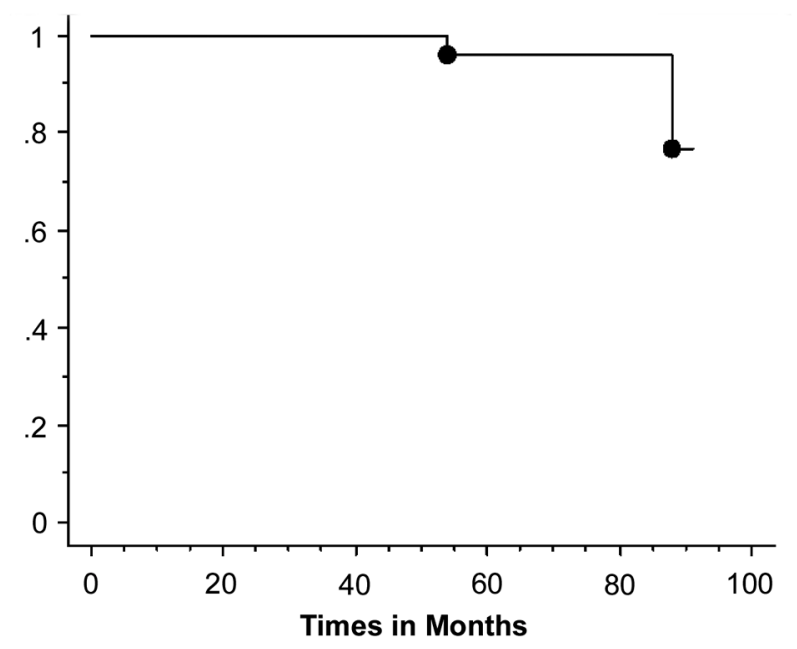

Figure 2 - Cumulative survival rate in patients undergoing nephron-sparing surgery by simple enucleation.

renal remnant. Uzzo \& Novick reviewed more than 1,800 cases of NSS in several large series and showed that the true biological significance of multicentric renal tumors and its implications for NSS remain to be completely elucidated (2). The major practical concern is to avoid the risk of positive margins after NSS. It was widely accepted that tumor should be excised with a piece of normal parenchyma however the minimal size of the parenchyma has been the subject of controversies for a long time. In the 1950s Vermooten first suggested that peripheral renal neoplasms could be excised leaving a margin of normal parenchyma around the tumor. He suggested the margin of at least $1 \mathrm{~cm}(12)$. For many years there was an agreement that $1 \mathrm{~cm}$ margin of normal parenchyma was the safest way to prevent local recurrence after NSS. For a variety of other tumors (e.g. colon cancer, breast cancer, melanoma, sarcoma) $1-2 \mathrm{~cm}$ margin is recommended to minimize the risk of local recurrence, which seems suitable due to the infiltrative growth of these tumors. RCC tends to compress normal parenchyma and forms a pseudocapsule around the tumor, thus the necessity of $1 \mathrm{~cm}$ resection margin seems to be overestimated. However, the rationale to perform NSS in patients with normal renal function and contralateral kidney is to preserve the renal parenchyma as much as possible and reduce the likelihood of deterioration of the renal function.
In recent years, the necessity of a conventional $1 \mathrm{~cm}$ margin has been revised substantially. Li et al. evaluated prospectively $82 \mathrm{RCC}$ of $4 \mathrm{~cm}$ or less resected by radical nephrectomy. The maximal extrapseudocapsule cancer extension was measured. Positive cancer lesion beyond the pseudocapsule was detected in $19.5 \%$ of cases with an average distance of $0.5 \mathrm{~mm}$ from the primary tumor. The authors considered that when partial nephrectomy is performed for $\mathrm{RCC}$ of $4 \mathrm{~cm}$ or less a $1 \mathrm{~cm}$ margin might be too much while enucleation alone may be associated with a significant risk of incomplete excision. Five $\mathrm{mm}$ margin could be enough to prevent possible local recurrences (13). Moreover, Pipper et al. showed that even $1 \mathrm{~mm}$ margin of normal tissue around the tumor is sufficient to prevent local recurrences. In these series by Piper et al., among the tumors resected with less than $1 \mathrm{~mm}$ margins no local recurrences were observed (14). Castilla et al. did not reveal any correlation between the size of the resection margin and disease progression (15). Sutharland et al. mentioned that the size of the margin was irrelevant as long as the surgical bed was free of residual tumor. Therefore, only a minimal margin of normal renal parenchyma must be removed during NSS for low stage RCC (16). Puppo et al. assessed safety and effectiveness of the excision of small renal cancer surrounded by a minimal layer of grossly normal parenchyma. None of the patients in these series had positive surgical margins nor had a local recurrence after surgery at median follow-up of 59 months (7). According to the authors, the mean and median shortest distances from the tumor to inked healthy tissue were $2.4 \mathrm{~mm}$ and $1.9 \mathrm{~mm}$ respectively. Regrettably, the authors did not provide data about the range of shortest distances from tumor to the inked healthy tissue. Therefore, we do not know whether or not in some of its enucleo-resections the tumor was resected without a rim of normal parenchyma.

All these reports suggest that incidentally detected small renal tumors (less than $4 \mathrm{~cm}$ ) have a benign behavior and the conventional $1 \mathrm{~cm}$ resection margin of normal parenchyma could be abandoned without any significant oncological risk. On the background of these studies reevaluation of the efficacy of simple enucleation in the treatment of RCC seems reasonable. Few studies in the late 1980s and early 
1990s investigated the efficacy of simple enucleation for the treatment of small RCC and showed 5-year survival rates similar to partial nephrectomy in a selected group of patients. Tumor enucleation has been shown to be effective in providing intermediate cancer-free intervals in patients with peripheral lesions $(17,18)$. However, other authors reported increasing as compared with the partial nephrectomy incidence of local recurrences after simple enucleation $(19,20)$. We think, this can be explained by the blunt finger enucleation technique instead of sharp dissection and improper patient selection. Lerner et al. showed that cause-specific survival was not significantly different after enucleation, in situ partial nephrectomy, or radical nephrectomy in patients with a solitary, $\leq 4$ $\mathrm{cm}$ tumors. They suggested that tumor enucleation using sharp dissection guided by the intraoperative frozen section analysis virtually eliminates the chance for incomplete primary tumor removal (6). Lapini et al. assessed the feasibility and effectiveness of simple enucleation for the elective treatment of RCC. They presented a retrospective study, which is based on the review of 107 clinical cases. Three patients had disease progression: 2 had local, 1 isolated and 1 associated recurrence with distant metastases. The authors show that simple tumor enucleation is a useful and acceptable approach for elective NSS (9). One of the major concern related with an enucleation during RCC is a possible microscopic tumor penetration of the pseudocapsule that surrounds the neoplasm but as it was reported by Li et al. (13) the average distance of tumor invasion beyond pseudocapsule is only $0.5 \mathrm{~mm}$, while in our patients tumor enucleation is always followed by coagulation of the enucleation bed, which provides approximately $1 \mathrm{~mm}$ thickness of parenchymal coagulation and therefore prevents risk of local recurrence. Despite of some similarity with enucleoresection we consider that enucleation with a coagulation of the tumor bed is different technique because it leads to the necrosis of $1 \mathrm{~mm}$ of healthy parenchyma while during the enucleo-resection the mean size of "sacrificed" parenchyma is about of $2.4 \mathrm{~mm}$ (7).

To our knowledge, there is no definite evidence of theoretical advantage of true partial nephrectomy over enucleation today. In our study we did not observe local recurrence during a long follow-up period. We had cancer specific and recurrence-free survival rates comparable to other published series $(2,4,5)$. Another important concern in NSS is the possibility of early postoperative complications. The rate of acute or delayed hemorrhage ranges between 1.3 $7.9 \%$ in published series (2). We did not observe these complications in our study. Urinary fistula is one of the most common complication after NSS with a reported mean rate of $6.5 \%$ (2). We consider that a minimal rate of morbidity in our study was caused partly due to strict patient selection and partly due to the technique of enucleation.

Finally, the aim of our study was not to show any advantages of simple enucleation over enucleoresection or wedge resection, but to try to demonstrate that oncological results of simple enucleation are at least not inferior comparing to other forms of NSS. At the same time enucleation leads to lower complication rate with maximal preservation of renal parenchyma.

\section{CONCLUSIONS}

The results of our study show that simple enucleation is an effective and acceptable method of operative treatment of RCC, which does not compromise the oncological outcome providing excellent long-term progression-free and cancer specific survival. It provides maximal preservation of renal parenchyma and lower incidence of postoperative complications. Larger and long-term studies are needed to prove improvement of the renal function after simple enucleation as compared to other NSS operations.

\section{CONFLICT OF INTEREST}

None declared.

\section{REFERENCES}

1. Herr HW: A history of partial nephrectomy for renal tumors. J Urol. 2005; 173: 705-8. 
2. Uzzo RG, Novick AC: Nephron sparing surgery for renal tumors: indications, techniques and outcomes. J Urol. 2001; 166: 6-18.

3. Russo P: Renal cell carcinoma: presentation, staging, and surgical treatment. Semin Oncol. 2000; 27: 16076.

4. Fergany AF, Hafez KS, Novick AC: Long-term results of nephron sparing surgery for localized renal cell carcinoma: 10-year follow-up. J Urol. 2000; 163: 442-5.

5. Herr HW: Partial nephrectomy for unilateral renal carcinoma and a normal contralateral kidney: 10-year follow-up. J Urol. 1999; 161: 33-4.

6. Lerner SE, Hawkins CA, Blute ML, Grabner A, Wollan PC, Eickholt JT, et al.: Disease outcome in patients with low stage renal cell carcinoma treated with nephron sparing or radical surgery. 1996. J Urol. 2002; 167: 884-9.

7. Puppo P, Introini C, Calvi P, Naselli A: Long term results of excision of small renal cancer surrounded by a minimal layer of grossly normal parenchyma: review of 94 cases. Eur Urol. 2004; 46: 477-81.

8. Franks ME, Hrebinko RL, Konety BR: Surgical enucleation for the treatment of renal tumors. Urol Int. 2003; 71: 184-9.

9. Lapini A, Serni S, Minervini A, Masieri L, Carini M: Progression and long-term survival after simple enucleation for the elective treatment of renal cell carcinoma: experience in 107 patients. J Urol. 2005; 174: 57-60.

10. Sobin LH, Wittekind CH (eds.): TNM Classification of Malignant Tumours. 6th ed. New York, Wiley-Liss Inc. 2002; vol. 6, pp. 193.
11. Fuhrman SA, Lasky LC, Limas C: Prognostic significance of morphologic parameters in renal cell carcinoma. Am J Surg Pathol. 1982; 6: 655-63.

12. Vermooten $\mathrm{V}$ : Indications for conservative surgery in certain renal tumors: a study based on the growth pattern of the cell carcinoma. J Urol. 1950; 64: 200-8.

13. Li QL, Guan HW, Zhang QP, Zhang LZ, Wang FP, Liu YJ: Optimal margin in nephron-sparing surgery for renal cell carcinoma $4 \mathrm{~cm}$ or less. Eur Urol. 2003; 44: 448-51.

14. Piper NY, Bishoff JT, Magee C, Haffron JM, Flanigan $\mathrm{RC}$, Mintiens A, et al.: Is a 1-CM margin necessary during nephron-sparing surgery for renal cell carcinoma? Urology. 2001; 58: 849-52.

15. Castilla EA, Liou LS, Abrahams NA, Fergany A, Rybicki LA, Myles J, et al.: Prognostic importance of resection margin width after nephron-sparing surgery for renal cell carcinoma. Urology. 2002; 60: 993-7.

16. Sutherland SE, Resnick MI, Maclennan GT, Goldman HB: Does the size of the surgical margin in partial nephrectomy for renal cell cancer really matter? J Urol. 2002; 167: 61-4.

17. Novick AC, Zincke H, Neves RJ, Topley HM: Surgical enucleation for renal cell carcinoma. J Urol. 1986; 135: 235-8.

18. Stephens R, Graham SD Jr: Enucleation of tumor versus partial nephrectomy as conservative treatment of renal cell carcinoma. Cancer. 1990; 65: 2663-7.

19. Marshall FF, Taxy JB, Fishman EK, Chang R: The feasibility of surgical enucleation for renal cell carcinoma. J Urol. 1986; 135: 231-4.

20. Blackley SK, Ladaga L, Woolfitt RA, Schellhammer PF: Ex situ study of the effectiveness of enucleation in patients with renal cell carcinoma. J Urol. 1988; 140: 6-10.

\footnotetext{
Correspondence address:

Dr. Ambrosi Pertia

Tsinandali str 9

0144, Tbilisi, Georgia

Fax: + $99532774-495$

E-mail: apertia@yahoo.com
} 


\section{EDITORIAL COMMENT}

Pertia \& Managadze performed a sharp dissection of small to medium sized renal tumors using a standard open technique in 30 patients. They termed their procedure simple enucleation although I had the impression that enucleation was performed with a finger to define by tactile means the true or pseudocapsule between the tumor and the normal parenchyma. They obtained a frozen section of the base however; they did not wait for the result to close the defect. They state that the results were always negative thus; they did not perform a total nephrectomy in any case because of a positive margin. Importantly although their series is relatively small, there were very few complications and the one leak was readily corrected with a stent. Although not stated it is likely all patients had a normal contralateral kidney and thus not surprisingly the renal function was not altered in any patient.

I perform the procedure in virtually an identical fashion as described by the authors. I believe an open flank approach for a partial nephrectomy for larger renal masses is a very safe procedure. The surgeon has excellent control of the vasculature and there is minimal bleeding. The new hemostatic agents are a real advance and provide an additional means to ensure hemostasis after careful suturing of small vessels before the capsular closure with gelfoam pledgets.
There is a word of caution however. Despite the nice results presented here given enough cases there might be the occasional patient who has a positive margin on a permanent section and the surgeon and his patient have the difficult decision of whether to return to the operating room (which usually means a nephrectomy) or monitor the kidney with lifelong imaging under the constant fear of a recurrence. It is always more comfortable to have that extra little bit of normal tissue around the tumor with a pathology report confirming that none of this tissue has any tumor. Do I always follow this rule? Not always but I feel more comfortable when I do. Once again, with appropriate vascular control and the new agents to aid in hemostasis the morbidity is not increased with this extra bit of caution. Given the "benign" behavior of most of these small tumors, one is not likely to tell a difference between no additional margin and a few mm of additional parenchyma. Some of our patients as in this series are young and in the case of a final permanent margin in such a patient, it will require many years to determine whether tumor was indeed left and the implications.

Finally, Webster's dictionary defines enucleate as "to remove without cutting in to". Thus, the authors must come up with another term since their dissection is sharp and they are cutting the parenchyma to remove the tumor.

Dr. Mark S. Soloway Professor and Chairman, Dept of Urology Miller Sch of Medicine, University of Miami

Miami, Florida, USA

E-mail:msoloway@miami.edu

\section{EDITORIAL COMMENT}

Although radical nephrectomy $(\mathrm{RN})$, as described by Robson et al. has been the gold standard for patients with renal cell carcinoma (RCC) for many decades, there is a trend towards nephron sparing surgery - NSS $(1,2)$. This evolution is the result of improved surgical technique, standardized staging, 
advanced radiological imaging and downward migration toward the diagnoses of asymptomatic, incidental, smaller, lower-stage lesions and the associated low rate of adrenal and lymph node metastases (3). NSS for tumors less than $4 \mathrm{~cm}$ (T1a) is at present widely accepted even for elective indications, while the use of NSS for patients with larger T1 tumors (T1b) is much more controversial (4). Although oncological results appear to be equal for NSS and RN for small tumors and the advantage is renal function is evident, NSS is still largely underused, as shown in a recent analysis (5). A margin of $1 \mathrm{~cm}$ of normal appearing parenchyma was long considered the standard in NSS (6). However, wide margins may compromise the residual renal function. The optimal resection margin is still debated because satellite lesions can be found more than $1.0 \mathrm{~cm}$ beyond the primary tumor (7). Sutherland et al. investigated the effects of surgical margin on recurrence. They concluded that the margin width is irrelevant if the tumor is completely resected and that it was not correlated with disease progression. The oncological result was independent of the margin width (8). Lapini et al. even showed that simple enucleation is a safe and acceptable approach (9). The present paper by Pertia et al. nicely provides additional evidence in favor of simple tumor enucleation in cT1a and cT1b RCC. In their limited series of 30 patients, complication rate was very low, with only 1 urinary leakage, requiring a double-J stent, and no hemorrhagic complications. The median follow-up was a substantial 71 months. Five- and 7-year cancer specific survival was impressive at $100 \%$ and $96.5 \%$ respectively. Of note is that they routinely performed frozen sections of the tumor bed, and patients who presented with a positive section margin underwent subsequent radical nephrectomy and were excluded from the analysis. This might have biased the results towards a more favorable outcome. It would have been interesting to know exactly how many patients were in this case. Furthermore, all resection beds were routinely coagulated, using diathermy spray coagulation. This is important, as coagulation will destroy another 1 to $2 \mathrm{~mm}$ rim of parenchyma. Lapini et al. similarly used diathermy spray coagulation or argon beam laser to the tumor bed. In our opinion, this might explain why enucleation provides the same results as enucleoresection, where a minimal rim of healthy tissue is resected together with the tumor (9).

\section{REFERENCES}

1. Robson CJ: Radical nephrectomy for renal cell carcinoma. J Urol. 1963; 89: 37-41.

2. Hafez KS, Novick AC, Campbell SC: Patterns of tumor recurrence and guidelines for follow-up after nephron sparing surgery for sporadic renal cell carcinoma. J Urol 1997; 157: 2067-70.

3. Goethuys H, Van Poppel H, Oyen R, Baert L: The case against fine-needle aspiration cytology for small solid kidney tumors. Eur Urol. 1996; 29: 284-7.

4. Campbell SC, Novick AC: Expanding the indications for elective partial nephrectomy: is this advisable? Eur Urol. 2006; 49: 952-4.

5. Miller DC, Hollingsworth JM, Hafez KS Daignault S, Hollenbeck BK: Partial nephrectomy for small renal masses: an emerging quality of care concern? J Urol. 2006; 175:853-8.

6. Novick AC: Surgery of the Kidney. In: Walsh P (ed.), Campbells Urology, vol. IV, 8th ed. Philadelphia, Saunders. 2002, pp. 3571.

7. Li QL, Guan HW, Zhang QP, Zhang LZ, Wang FP, Liu YJ: Optimal margin in nephron-sparing surgery for renal call carcinoma $4 \mathrm{~cm}$ or less. Eur Urol. 2003; 44: 448-51.

8. Sutherland SE, Resnick MI, Maclennan GT, Goldman HB: Does the size of the surgical margin in partial nephrectomy for renal cell cancer really matter? J Urol. 2002; 167:61-4.

9. Lapini A, Serni S, Minervini A, Masieri L, Carini M: Progression and long-term survival after simple enucleation for the elective treatment of renal cell carcinoma: experience in 107 patients. J Urol. 2005; 174: 57-60.

Dr. Steven Joniau \& Dr. Hein Van Poppel Department of Urology University Hospital Leuven Leuven, Belgium E-mail: steven.joniau@uz.kuleuven.ac.be 